\title{
UNIVERSAL QUANTUM COMPUTING AND THREE-MANIFOLDS
}

\author{
MICHEL PLANAT $\dagger$, RAYMOND ASCHHEIM $\ddagger$ \\ MARCELO M. AMARAL $\ddagger$ AND KLEE IRWIN $\ddagger$
}

\begin{abstract}
A single qubit may be represented on the Bloch sphere or similarly on the 3-sphere $S^{3}$. Our goal is to dress this correspondence by converting the language of universal quantum computing (uqc) to that of 3-manifolds. A magic state and the Pauli group acting on it define a model of uqc as a POVM that one recognizes to be a 3-manifold $M^{3}$. E. g., the $d$-dimensional POVMs defined from subgroups of finite index of the modular group $P S L(2, \mathbb{Z})$ correspond to $d$-fold $M^{3}$ - coverings over the trefoil knot. In this paper, one also investigates quantum information on a few 'universal' knots and links such as the figure-of-eight knot, the Whitehead link and Borromean rings, making use of the catalog of platonic manifolds available on SnapPy. Further connections between POVMs based uqc and $M^{3}$ 's obtained from Dehn fillings are explored.
\end{abstract}

PACS: 03.67.Lx, 03.65.Wj, 03.65.Aa, 02.20.-a, 02.10.Kn, 02.40.Pc, 02.40.Sf MSC codes: 81P68, 81P50, 57M25, 57R65, 14H30, 20E05, 57M12

Keywords: quantum computation, IC-POVMs, knot theory, three-manifolds, branch coverings, Dehn surgeries.

Manifolds are around us in many guises.

As observers in a three-dimensional world, we are most familiar with twomanifolds: the surface of a ball or a doughnut or a pretzel, the surface of a house or a tree or a volleyball net...

Three-manifolds may be harder to understand at first. But as actors and movers in a three-dimensional world, we can learn to imagine them as alternate universes.

\section{INTRODUCTION}

Mathematical concepts pave the way for improvements in technology. As far as topological quantum computation is concerned, non abelian anyons have been proposed as an attractive (fault tolerant) alternative to standard quantum computing which is based on a universal set of quantum gates [5, 6, 7, 8. Anyons are two-dimensional quasiparticles with world lines forming braids in space-time. Whether non abelian anyons do exist in the real world and/or would be easy to create artificially is still open to discussion. Three-dimensional topological quantum computing beyond anyons [9] still is not well developed although, as it will be shown in this essay, it is a straightforward consequence of a set of ideas belonging to standard universal quantum computation (uqc) and simultaneously to three-dimensional topology. For quantum computation, we have in mind the concepts of magic states and the related positive-operator valued measures (POVMs) that were investigated in detail in [10, 11, 12]. For three-dimensional topology, the 
starting point consists of the Poincaré and Thurston conjectures, now theorems [1]. Three-dimensional topological quantum computing would federate the foundations of quantum mechanics and cosmology, a recurrent dream of many physicists. Three-dimensional topology was already investigated by several groups in the context of quantum information [13, 14, high energy physics [15, 16], biology [17] and consciousness studies [18].

1.1. From Poincaré conjecture to uqc. Poincaré conjecture is the elementary (but deep) statement that every simply connected, closed 3-manifold is homeomorphic to the 3 -sphere $S^{3}$ [19]. Having in mind the correspondence between $S^{3}$ and the Bloch sphere that houses the qubits $\psi=a|0\rangle+b|1\rangle$, $a, b \in \mathbb{C},|a|^{2}+|b|^{2}=1$, one would desire a quantum translation of this statement. For doing this, one may use the picture of the Riemann sphere $\mathbb{C} \cup \infty$ in parallel to that of the Bloch sphere and follow F. Klein lectures on the icosahedron to perceive the platonic solids within the landscape [20]. This picture fits well the Hopf fibrations [21, their entanglements described in [22, 23] and quasicrystals [25, 24]. But we can be more ambitious and dress $S^{3}$ in an alternative way that reproduces the historic thread of the proof of Poincaré conjecture. Thurston's geometrization conjecture, from which Poincaré conjecture follows, dresses $S^{3}$ as a 3-manifold not homeomorphic to $S^{3}$. The wardrobe of 3 -manifolds $M^{3}$ is huge but almost every dress is hyperbolic and W. Thurston found the recipes for them [1]. Every dress is identified thanks to a signature in terms of invariants. For our purpose, the fundamental group $\pi_{1}$ of $M^{3}$ does the job.

The three-dimensional space surrounding a knot $K$-the knot complement $S^{3} \backslash K$ - is an example of a three-manifold [1, 26]. We will be especially interested by the trefoil knot that underlies work of the first author [2] as well as the figure-of-eight knot, the Whitehead link and the Borromean rings because they are universal (in a sense described below), hyperbolic and allows to build 3-manifolds from platonic manifolds [4]. Such manifolds carry a quantum geometry corresponding to quantum computing and (possibly informationally complete: IC) POVMs identified in our earlier work [2, 11, 12 .

According to [3, the knot $K$ and the fundamental group $G=\pi_{1}\left(S^{3} \backslash K\right)$ are universal if every closed and oriented 3-manifold $M^{3}$ is homeomorphic to a quotient $\mathbb{H} / G$ of the hyperbolic 3 -space $\mathbb{H}$ by a subgroup $H$ of finite index $d$ of $G$. The figure-of-eight knot and the Whitehead link are universal. The catalog of the finite index subgroups of their fundamental group $G$ and of the corresponding 3-manifolds defined from the $d$-fold coverings [27] can easily been established up to degree 8, using the software SnapPy [28].

In paper [2] of the first author, it has been found that minimal $d$-dimensional IC-POVMs (sometimes called MICs) may be built from finite index subgroups of the modular group $\Gamma=P S L(2, \mathbb{Z}$ ). To such an IC (or MIC) is associated a subgroup of index $d$ of $\Gamma$, a fundamental domain in the Poincaré upper-half plane and a signature in terms of genus, elliptic points and cusps as summarized in [2, Fig. 1]. There exists a relationship between the modular group $\Gamma$ and the trefoil knot $T_{1}$ since the fundamental group $\pi_{1}\left(S^{3} \backslash T_{1}\right)$ of the knot complement is the braid group $B_{3}$, the central extension of $\Gamma$. 


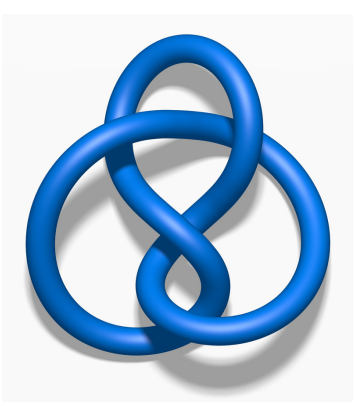

(a)

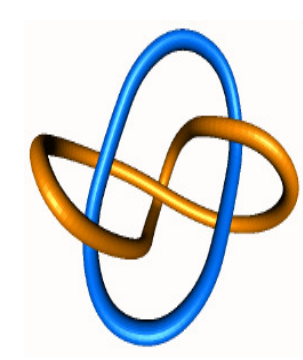

(b)

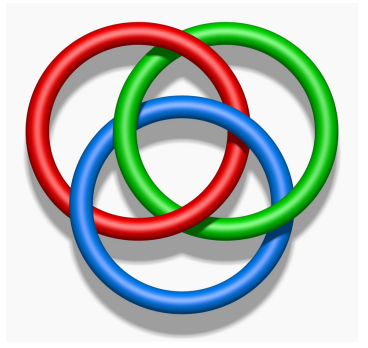

(c)

Figure 1. (a) The figure-of-eight knot: $K 4 a 1=$ otet $02_{00001}=m 004, \quad(\mathrm{~b})$ the Whitehead link

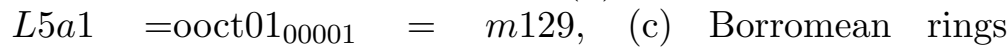
$L 6 a 4=$ ooct $02_{00005}=t 12067$.

But the trefoil knot and the corresponding braid group $B_{3}$ are not universal [29] which forbids the relation of the finite index subgroups of $B_{3}$ to all three-manifolds.

It is known that two coverings of a manifold $M$ with fundamental group $G=\pi_{1}(M)$ are equivalent if there exists a homeomorphism between them. Besides, a $d$-fold covering is uniquely determined by a subgroup of index $d$ of the group $G$ and the inequivalent $d$-fold coverings of $M$ correspond to conjugacy classes of subgroups of $G$ [27]. In this paper we will fuse the concepts of a three-manifold $M^{3}$ attached to a subgroup $H$ of index $d$ and the POVM, possibly informationally complete (IC), found from $H$ (thanks to the appropriate magic state and related Pauli group factory).

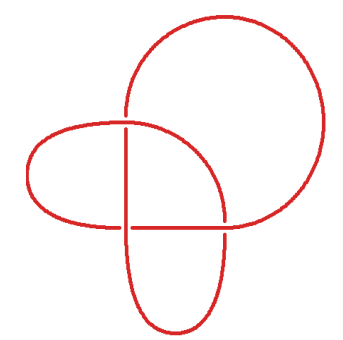

(a)

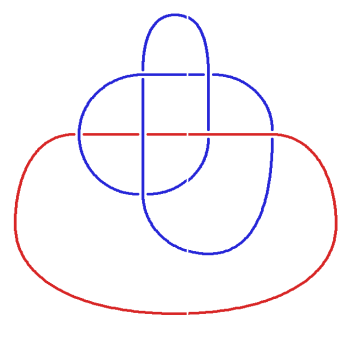

(b)

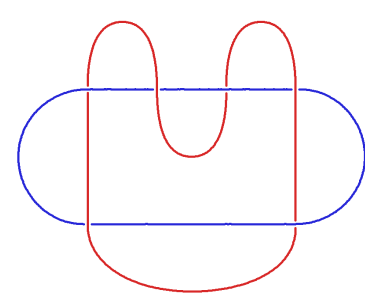

(c)

Figure 2. (a) The trefoil knot $T_{1}=K 3 a 1=3_{1}$, (b) the link $L 7 n 1$ associated to the Hesse SIC, (c) the link L6a3 associated to the two-qubit IC. 
MICHEL PLANAT $\dagger$, RAYMOND ASCHHEIM $\ddagger$, MARCELO M. AMARAL $\ddagger$ AND KLEE IRWIN $\ddagger$

1.2. Minimal informationally complete POVMs and uqc. In our approach [12, 2, minimal informationally complete (IC) POVMs are derived from appropriate fiducial states under the action of the (generalized) Pauli group. The fiducial states also allow to perform universal quantum computation [11.

A POVM is a collection of positive semi-definite operators $\left\{E_{1}, \ldots, E_{m}\right\}$ that sum to the identity. In the measurement of a state $\rho$, the $i$-th outcome is obtained with a probability given by the Born rule $p(i)=\operatorname{tr}\left(\rho E_{i}\right)$. For a minimal IC-POVM (or MIC), one needs $d^{2}$ one-dimensional projectors $\Pi_{i}=\left|\psi_{i}\right\rangle\left\langle\psi_{i}\right|$, with $\Pi_{i}=d E_{i}$, such that the rank of the Gram matrix with elements $\operatorname{tr}\left(\Pi_{i} \Pi_{j}\right)$, is precisely $d^{2}$. A SIC-POVM (the $S$ means symmetric) obeys the relation $\left|\left\langle\psi_{i} \mid \psi_{j}\right\rangle\right|^{2}=\operatorname{tr}\left(\Pi_{i} \Pi_{j}\right)=\frac{d \delta_{i j}+1}{d+1}$, that allows the explicit recovery of the density matrix as in [30, eq. (29)].

New minimal IC-POVMs (i.e. whose rank of the Gram matrix is $d^{2}$ ) and with Hermitian angles $\left|\left\langle\psi_{i} \mid \psi_{j}\right\rangle\right|_{i \neq j} \in A=\left\{a_{1}, \ldots, a_{l}\right\}$ have been discovered 2]. A SIC is equiangular with $|A|=1$ and $a_{1}=\frac{1}{\sqrt{d+1}}$. The states encountered are considered to live in a cyclotomic field $\mathbb{F}=\mathbb{Q}\left[\exp \left(\frac{2 i \pi}{n}\right)\right]$, with $n=\mathrm{GCD}(d, r)$, the greatest common divisor of $d$ and $r$, for some $r$. The Hermitian angle is defined as $\left|\left\langle\psi_{i} \mid \psi_{j}\right\rangle\right|_{i \neq j}=\left\|\left(\psi_{i}, \psi_{j}\right)\right\|^{\frac{1}{\operatorname{deg}}}$, where $\|\cdot\|$ means the field norm of the pair $\left(\psi_{i}, \psi_{j}\right)$ in $\mathbb{F}$ and deg is the degree of the extension $\mathbb{F}$ over the rational field $\mathbb{Q}[12]$.

The fiducial states for SIC-POVMs are quite difficult to derive and seem to follow from algebraic number theory [31].

Except for $d=3$, the IC-POVMs derived from permutation groups are not symmetric and most of them can be recovered thanks to subgroups of index $d$ of the modular group $\Gamma$ [2, Table 2]. The geometry of the qutrit Hesse SIC is shown in Fig 3a. It follows from the action of the qutrit Pauli group on magic/fiducial states of type $(0,1, \pm 1)$. For $d=4$, the action of the two-qubit Pauli group on the magic/fiducial state of type $\left(0,1,-\omega_{6}, \omega_{6}-1\right)$ with $\omega_{6}=\exp \left(\frac{2 i \pi}{6}\right)$ results into a minimal IC-POVM whose geometry of triple products of projectors $\Pi_{i}$ turns out to correspond to the commutation graph of Pauli operators, see Fig. 3b and [2, Fig. 2]. For $d=5$, the geometry of an IC consists of copies of the Petersen graph reproduced in Fig. 3c. For $d=6$, the geometry consists of components looking like Borromean rings (see [2, Fig. 2] and Table 1 below).

While $\Gamma$ serves as a motivation for investigating the trefoil knot manifold in relation to uqc and the corresponding ICs, it is important to put the uqc problem in the wider frame of Poincaré conjecture, the Thurston's geometrization conjecture and the related 3-manifolds. E.g. ICs may also follow from hyperbolic or Seifert 3-manifolds as shown in tables 2 to 5 of this paper.

1.3. Organization of the paper. The paper runs as follows. Sec. 2 deals about the relationship between quantum information seen from the modular group $\Gamma$ and from the trefoil knot 3-manifold. Sec. 3 deals about the (platonic) 3-manifolds related to coverings over the figure-of-eight knot, Whitehead link and Borromean rings, and how they relate to minimal ICPOVMs. Sec. 4 describes the important role played by Dehn fillings for 


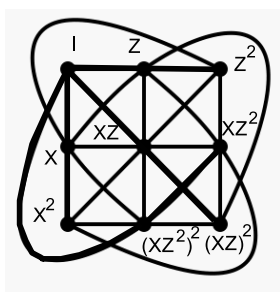

The Hesse SIC for qutrits fiducials : $(0,1,1)$ or $(0,1,-1)$

(a)

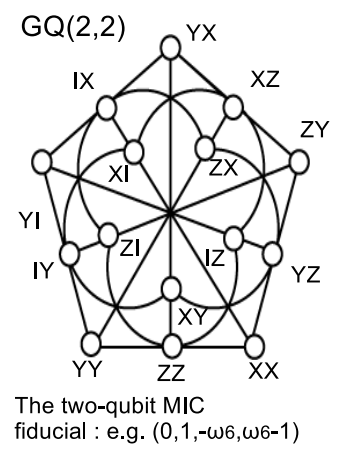

(b)

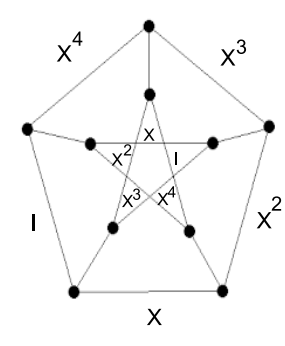

component of a 5-dit IC-POVM fiducial : e.g. $(0,-1,-1,1)$

(c)

FIGURE 3. Geometrical structure of low dimensional MICs: (a) the qutrit Hesse SIC, (b) the two-qubit MIC that is the generalized quadrangle of order two $G Q(2,2)$, (c) the basic conponent of the 5-dit MIC that is the Petersen graph. The coordinates on each diagram are the $d$-dimensional Pauli operators that act on the fiducial state, as shown.

describing the many types of 3 -manifolds that may relate to topological quantum computing.

\section{QUANTUM INFORMATION FROM THE MODUlaR GROUP $\Gamma$ AND THE RELATED TREFOIL KNOT $T_{1}$}

In this section, we describe the results established in [2] in terms of the 3manifolds corresponding to coverings of the trefoil knot complement $S^{3} \backslash T_{1}$.

Let us introduce to the group representation of a knot complement $\pi_{1}\left(S^{3} \backslash\right.$ $K)$. A Wirtinger representation is a finite representation of $\pi_{1}$ where the relations are the form $w g_{i} w^{-1}=g_{j}$ where $w$ is a word in the $k$ generators $\left\{g_{1}, \cdots, g_{k}\right\}$. For the trefoil knot $T_{1}=K 3 a 1=3_{1}$ shown in Fig. 2a, a Wirtinger representation is 32

$$
\pi_{1}\left(S^{3} \backslash T_{1}\right)=\langle x, y \mid y x y=x y x\rangle \text { or equivalently } \pi_{1}=\left\langle x, y \mid y^{2}=x^{3}\right\rangle .
$$

In the rest of the paper, the number of $d$-fold coverings of the manifold $M^{3}$ corresponding to the knot $T$ will be displayed as the ordered list $\eta_{d}(T)$, $d \in\{1 . .10 \ldots\}$. For $T_{1}$ it is

$$
\eta_{d}\left(T_{1}\right)=\{1,1,2,3,2,8,7,10,18,28, \ldots\} .
$$

Details about the corresponding $d$-fold coverings are in Table 1. As expected, the coverings correspond to subgroups of index $d$ of the fundamental group associated to the trefoil knot $T_{1}$.

2.0.1. Cyclic branched coverings over the trefoil knot. Let $p, q, r$ be three positive integers (with $p \leq q \leq r$ ), the Brieskorn 3-manifold $\Sigma(p, q, r)$ is the intersection in $\mathbb{C}^{3}$ of the 5 -sphere $S^{5}$ with the surface of equation $z_{1}^{p}+z_{2}^{q}+$ $z_{3}^{r}=1$. In [34, it is shown that a $r$-fold cyclic covering over $S^{3}$ branched along a torus knot or link of type $(p, q)$ is a Brieskorn 3-manifold $\Sigma(p, q, r)$ 
MICHEL PLANAT $\dagger$, RAYMOND ASCHHEIM $\ddagger$, MARCELO M. AMARAL $\ddagger$ AND KLEE IRWIN $\ddagger$

\begin{tabular}{|l|c|c|r|l|r|r||r|}
\hline \hline $\mathrm{d}$ & ty & hom & cp & gens & CS & link & type in [2] \\
\hline 2 & cyc & $\frac{1}{3}+1$ & 1 & 2 & $-1 / 6$ & & \\
\hline 3 & irr & $1+1$ & 2 & 2 & $1 / 4$ & L7n1 & $\Gamma_{0}(2)$, Hesse SIC \\
. & cyc & $\frac{1}{2}+\frac{1}{2}+1$ & 1 & 3 &. & & $A_{4}$ \\
\hline 4 & irr & $1+1$ & 2 & 2 & $1 / 6$ & L6a3 & $\Gamma_{0}(3), 2 \mathrm{QB} \mathrm{IC}$ \\
. & irr & $\frac{1}{2}+1$ & 1 & 3 &. & & $4 A^{0}, 2 \mathrm{QB}-\mathrm{IC}$ \\
. & cyc & $\frac{1}{3}+1$ & 1 & 2 &. & & $S_{4}$ \\
\hline 5 & cyc & 1 & 1 & 2 & $5 / 6$ & & $A_{5}$ \\
. & irr & $\frac{1}{3}+1$ & 1 & 3 &. & & $5 A^{0}, 5$-dit IC \\
\hline 6 & reg & $1+1+1$ & 3 & 3 & 0 & L6n1 & $\Gamma(2), 6$-dit IC \\
. & cyc & $1+1+1$ & 1 & 3 &. & L6n1 & $\Gamma^{\prime}, 6$-dit IC \\
. & irr & $1+1+1$ & 3 & 3 &. & & \\
. & irr & $\frac{1}{2}+1+1$ & 2 & 3 &. & & $3 C^{0}, 6$-dit IC \\
. & irr & $\frac{1}{2}+1+1$ & 2 & 3 &. & & $\Gamma_{0}(4), 6$-dit IC \\
. & irr & $\frac{1}{2}+1+1$ & 2 & 3 &. & & $\Gamma_{0}(5), 6$-dit IC \\
. & irr & $\frac{1}{2}+\frac{1}{2}+\frac{1}{2}+1$ & 1 & 4 &. & & \\
. & irr & $\frac{1}{3}+\frac{1}{3}+1$ & 1 & 3 &. & & \\
\hline \hline 7 & cyc & 1 & 1 & 2 & $-5 / 6$ & & \\
. & irr & $1+1$ & 2 & 3 &. & & NC 7-dit IC \\
. & irr & $\frac{1}{2}+\frac{1}{2}+1$ & 1 & 4 &. & & $7 A^{0} 7$-dit IC \\
\hline 8 & irr & $1+1$ & 2 & 2 & $-1 / 6$ & & \\
. & cyc & $\frac{1}{3}+1$ & 2 & 2 &. & & \\
. & cyc & $\frac{1}{3}+1+1$ & 2 & 3 &. & & $8 A^{0}, \sim$ 8-dit IC \\
. & cyc & $\frac{1}{6}+1$ & 1 & 4 &. & & \\
\hline \hline
\end{tabular}

TABLE 1. Coverings of degree $d$ over the trefoil knot found from SnapPy [28]. The related subgroup of modular group $\Gamma$ and the corresponding IC-POVM [2] (when applicable) is in the right column. The covering is characterized by its type ty, homology group hom (where 1 means $\mathbb{Z}$ ), the number of cusps $\mathrm{cp}$, the number of generators gens of the fundamental group, the Chern-Simons invariant CS and the type of link it represents (as identified in SnapPy). The case of cyclic coverings corresponds to Brieskorn 3-manifolds as explained in the text: the spherical groups for these manifolds is given at the right hand side column.

(see also Sec 4.1). For the spherical case $p^{-1}+q^{-1}+r^{-1}>1$, the group associated to a Brieskorn manifold is either dihedral [that is the group $D_{r}$ for the triples $(2,2, r)$ ], tetrahedral [that is $A_{4}$ for $(2,3,3)$ ], octahedral [that is $S_{4}$ for $(2,3,4)$ ] or icosahedral [that is $A_{5}$ for $(2,3,5)$ ]. The Euclidean case $p^{-1}+q^{-1}+r^{-1}=1$ corresponds to $(2,3,6),(2,4,4)$ or $(3,3,3)$. The remaining cases are hyperbolic.

The cyclic branched coverings with spherical groups for the trefoil knot [which is of type $(2,3)$ ] are identified in right hand side column of Table 1.

2.0.2. Irregular branched coverings over the trefoil knot. The right hand side column of Table 1 shows the subgroups of $\Gamma$ identified in [2, Table 1] as 
corresponding to a minimal IC-POVM. Let us give a few more details how to attach a MIC to some coverings/subgroups of the trefoil knot fundamental group $\pi_{1}\left(T_{1}\right)$. Columns 1 to 6 in Table 1 contain information available in SnapPy [28], with $d$, ty, hom, cp, gens and CS the degree, the type, the first homology group, the number of cusps, the number of generators and the Chern-Simons invariant of the relevant covering, respectively. In column 7, a link is possibly identified by SnapPy when the fundamental group and other invariants attached to the covering correspond to those of the link. For our purpose, we also are interested in the possible recognition of a MIC behind some manifolds in the table.

For the irregular covering of degree 3 and first homology $\mathbb{Z}+\mathbb{Z}$, the fundamental group provided by SnapPy is $\pi_{1}\left(M^{3}\right)=\left\langle a, b \mid a b^{-2} a^{-1} b^{2}\right\rangle$ that of course corresponds to a representative $H$ of one of the two conjugacy classes of subgroups of index 3 of the modular group $\Gamma$, following the theory of [27]. The organization of cosets of $H$ in the two-generator group $G=$ $\left\langle a, b \mid a^{2}, y^{3}\right\rangle \cong \Gamma$ thanks to the Coxeter-Todd algorithm (implemented in Magma) results into the permutation group $P=\langle 3 \mid(1,2,3),(2,3)\rangle$, as in [2, Sec. 3.1]. This permutation group is also the one obtained from the congruence subgroup $\Gamma_{0}(2) \cong S_{3}$ of $\Gamma$ (where $S_{3}$ is the three-letter symmetric group) whose fundamental domain is in [2, Fig. 1b]. Then, the eigenstates of the permutation matrix in $S_{3}$ of type $(0,1, \pm 1)$ serve as magic/fiducial state for the Hesse SIC [2, 12].

A similar reasoning applied to the irregular coverings of degree 4 , and first homology $\mathbb{Z}+\mathbb{Z}$ and $\frac{\mathbb{Z}}{2}+\mathbb{Z}$ leads to the recognition of congruence subgroups $\Gamma_{0}(3)$ and $4 A^{0}$, respectively, behind the corresponding manifolds. It is known from [2, Sec. 3.2] that they allow the construction of two-qubit minimal IC-POVMs. For degree 5, the equiangular 5-dit MIC corresponds to the irregular covering of homology $\frac{\mathbb{Z}}{3}+\mathbb{Z}$ and to the congruence subgroup $5 A^{0}$ in $\Gamma$ (as in [2, Sec. 3.3]).

Five coverings of degree 6 allow the construction of the (two-valued) 6-dit IC-POVM whose geometry contain the picture of Borromean rings [2, Fig. 2c]. The corresponding congruence subgroups of $\Gamma$ are identified in table 1. The first two, viz $\Gamma(2)$ and $\Gamma^{\prime}$, define 3-manifolds whose fundamental group is the same than the one of the link $L 6 n 1$, alias the Borromean rings with surgeries of slope $(0,1)$ on two cusps (see Sect. 4 for more on this topic). The other three coverings leading to the 6 -dit IC are the congruence subgroups $3 C^{0}, \Gamma_{0}(4)$ and $\Gamma_{0}(5)$.

\section{QuANTUM INFORMation FROM UNIVERSAL KNOTS AND LINKS}

At the previous section, we found the opportunity to rewrite results about the existence and construction of $d$-dimensional MICs in terms of the threemanifolds corresponding to some degree $d$ coverings of the trefoil knot $T_{1}$. But neither $T_{1}$ nor the manifolds corresponding to its covering are hyperbolic. In the present section, we proceed with hyperbolic (and universal) knots and links and display the three-manifolds behind the low dimensional MICs. The method is as above in Sec. 2 in the sense that the fundamental group of a 3-manifold $M^{3}$ attached to a degree $d$-covering is the one of 
MICHEL PLANAT $\dagger$, RAYMOND ASCHHEIM $\ddagger$ MARCELO M. AMARAL $\ddagger$ AND KLEE IRWIN $\ddagger$

a representative of the conjugacy class of subgroups of the corresponding index in the relevant knot or link.

3.1. Three-manifolds pertaining to the figure-of-eight knot. The fundamental group for the figure-of-eight knot $K_{0}$ is

$$
\pi_{1}\left(S^{3} \backslash K_{0}\right)=\left\langle x, y \mid y * x * y^{-1} x y=x y x^{-1} y x\right\rangle .
$$

and the number of $d$-fold coverings is in the list

$$
\eta_{d}\left(K_{0}\right)=\{1,1,1,2,4,11,9,10,11,38, \ldots\} .
$$

Table 2 establishes the list of 3-manifolds corresponding to subgroups of index $d \leq 7$ of the universal group $G=\pi_{1}\left(S^{3} \backslash K_{0}\right)$. The manifolds are labeled otet $N_{n}$ in [4] because they are oriented and built from $N=2 d$ tetrahedra, with $n$ an index in the table. The identification of 3-manifolds of finite index subgroups of $G$ was first obtained by comparing the cardinality list $\eta_{d}(H)$ of the corresponding subgroup $H$ to that of a fundamental group of a tetrahedral manifold in SnapPy table [28]. But of course there is more straightforward way to perform this task by identifying a subgroup $H$ to a degree $d$ covering of $K_{0}$ [27]. The full list of $d$-branched coverings over the figure eight knot up to degree 8 is available in SnapPy. Extra invariants of the corresponding $M^{3}$ may be found there. In addition, the lattice of

\begin{tabular}{|c|c|c|c|c|c|c|}
\hline d & ty & $M^{3}$ & $\mathrm{cp}$ & $\mathrm{rk}$ & $\mathrm{pp}$ & comment \\
\hline 2 & cyc & otet $04_{00002}, m 206$ & 1 & 2 & & \\
\hline 3 & cyc & otet06 $00003, s 961$ & 1 & 3 & & \\
\hline 4 & $\begin{array}{l}\text { irr } \\
\text { cyc }\end{array}$ & $\begin{array}{c}\text { otet } 08_{00002}, L 10 n 46, t_{12840} \\
\text { otet } 08_{00007}, t 12839\end{array}$ & 1 & $\begin{array}{r}4 \\
16\end{array}$ & 1 & $\begin{array}{c}\text { Mom-4s [36] } \\
\text { 2-qubit IC }\end{array}$ \\
\hline 5 & $\begin{array}{l}\text { cyc } \\
\text { irr } \\
\text { irr }\end{array}$ & $\begin{array}{l}\text { otet } 10_{00019} \\
\text { otet } 10_{00006}, L 8 a 20 \\
\text { otet10 }\end{array}$ & $\begin{array}{l}1 \\
3 \\
2\end{array}$ & $\begin{array}{r}21 \\
15,21 \\
25\end{array}$ & 1 & 5-dit IC \\
\hline 6 & $\begin{array}{l}\text { cyc } \\
\text { irr } \\
\text { irr } \\
\text { irr } \\
\text { irr }\end{array}$ & $\begin{array}{c}\text { otet } 12_{00013} \\
\text { otet } 12_{00041} \\
\text { otet } 12_{00039}, \text { otet } 12_{00038} \\
\text { otet } 12_{00017} \\
\text { otet } 12_{00000}\end{array}$ & $\begin{array}{l}2 \\
1 \\
2 \\
2\end{array}$ & $\begin{array}{l}28 \\
36 \\
31 \\
33 \\
36\end{array}$ & 2 & 6 -dit IC \\
\hline 7 & $\begin{array}{l}\text { cyc } \\
\text { irr } \\
\text { irr }\end{array}$ & $\begin{array}{c}\text { otet } 14_{00019} \\
\text { otet14 } 4_{00002}, L 14 n 55217 \\
\text { otet } 14_{00035}\end{array}$ & 1 & $\begin{array}{l}43 \\
49 \\
49\end{array}$ & $\begin{array}{l}2 \\
2\end{array}$ & $\begin{array}{l}\text { 7-dit IC } \\
\text { 7-dit IC }\end{array}$ \\
\hline
\end{tabular}
branched coverings over $K_{0}$ was investigated in [35].

TABLE 2. Table of 3-manifolds $M^{3}$ found from subgroups of finite index $d$ of the fundamental group $\pi_{1}\left(S^{3} \backslash K_{0}\right)$ (alias the $d$-fold coverings of $K_{0}$ ). The terminology in column 3 is that of Snappy [28. The identified $M^{3}$ is made of $2 d$ tetrahedra and has cp cusps. When the rank $r k$ of the POVM Gram matrix is $d^{2}$ the corresponding IC-POVM shows $p p$ distinct values of pairwise products as shown. 
Let us give more details about the results summarized in Table 2. Using Magma, the conjugacy class of subgroups of index 2 in the fundamental group $G$ is represented by the subgroup on three generators and two relations as follows $H=\left\langle x, y, z \mid y^{-1} z x^{-1} z y^{-1} x^{-2}, z^{-1} y x z^{-1} y z^{-1} x y\right\rangle$, from which the sequence of subgroups of finite index can be found as $\eta_{d}\left(M^{3}\right)=$ $\{1,1,5,6,8,33,21,32, \cdots\}$. The manifold $M^{3}$ corresponding to this sequence is found in Snappy as otet04 00002 , alias $m 206$.

The conjugacy class of subgroups of index 3 in $G$ is represented as $H=\left\langle x, y, z \mid x^{-2} z x^{-1} y z^{2} x^{-1} z y^{-1}, z^{-1} x z^{-2} x z^{-2} y^{-1} x^{-2} z y\right\rangle$, with $\eta_{d}\left(M^{3}\right)=\{1,7,4,47,19,66,42,484, \cdots\}$ corresponding to the manifold otet06 00003 , alias $s 961$.

As shown in Table 2, there are two conjugacy classes of subgroups of index 4 in $G$ corresponding to tetrahedral manifolds otet $08_{00002}$ (the permutation group $P$ organizing the cosets is $\mathbb{Z}_{4}$ ) and otet08 $8_{00007}$ (the permutation group organizing the cosets is the alternating group $A_{4}$ ). The latter group/manifold has fundamental group

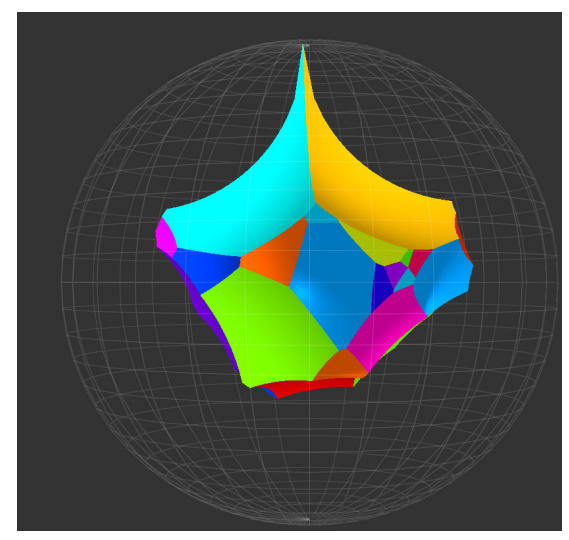

Manifold otet08_00007

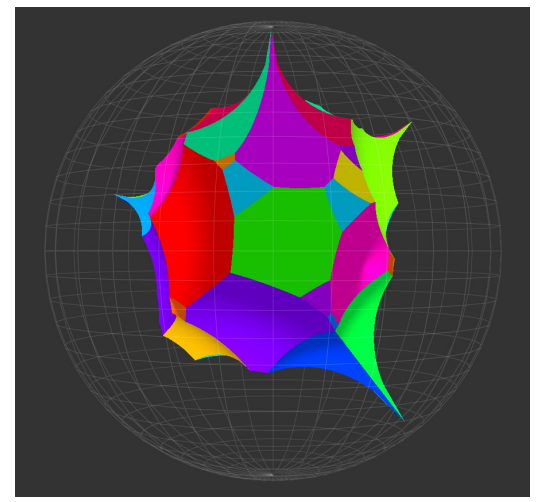

Manifold ooct04_00058

(a)

(b)

Figure 4. Two platonic three-manifolds leading to the construction of the two-qubit MIC. Details are given in Tables 2 and 3 .

$H=\left\langle x, y, z \mid y x^{-1} y^{-1} z^{-1} x y^{-2} x y z x^{-1} y, z x^{-1} y x^{-1} y x^{-1} z y x^{-1} y^{-1} z^{-1} x y^{-1}\right\rangle$, with cardinality sequences of subgroups as $\eta_{d}\left(M^{3}\right)=\{1,3,8,25,36,229,435 \cdots\}$. To $H$ is associated an IC-POVM [12, 2] which follows from the action of the two-qubit Pauli group on a magic/fiducial state of type $\left(0,1,-\omega_{6}, \omega_{6}-1\right)$, with $\omega_{6}=\exp (2 i \pi / 6)$ a six-root of unity.

For index 5, there are three types of 3-manifolds corresponding to the subgroups $H$. The tetrahedral manifold otet $10_{00026}$ of cardinality sequence $\eta_{d}\left(M^{3}\right)=\{1,7,15,88,123,802,1328 \cdots\}$, is associated to a 5 -dit equiangular IC-POVM, as in [12, Table 5]. 
MOICHEL PLANAT $\dagger$, RAYMOND ASCHHEIM $\ddagger$ MARCELO M. AMARAL $\ddagger$ AND KLEE IRWIN $\ddagger$

For index 6, the 11 coverings define six classes of 3-manifolds and two of them: otet $12_{00041}$ and otet $12_{00000}$ are related to the construction of ICs. For index 7, one finds three classes of 3-manifolds with two of them: otet $14_{00002}$ (alias $L 14 n 55217$ ) and otet $14_{00035}$ are related to ICs. Finally, for index 7, 3 types of 3-manifolds exist, two of them relying on the construction of the 7-dit (two-valued) IC. For index 8, there exists 6 distinct 3-manifolds (not shown) none of them leading to an IC.

\begin{tabular}{|c|c|c|c|c|c|c|}
\hline $\mathrm{d}$ & ty & $\bar{M}^{3}$ & cp & rk & $\mathrm{pp}$ & comment \\
\hline 2 & $\begin{array}{l}\text { cyc } \\
\text { cyc }\end{array}$ & $\begin{array}{l}\text { ooct } 02_{00003}, t 12066, L 8 n 5 \\
\quad \text { ooct02 } \\
00018, t 12048\end{array}$ & $\begin{array}{l}3 \\
2\end{array}$ & $\begin{array}{l}2 \\
2\end{array}$ & & $\begin{array}{l}\text { Mom-4s } 36 \\
\text { Mom-4s } 36\end{array}$ \\
\hline 3 & $\begin{array}{l}\text { cyc } \\
\text { cyc } \\
\text { irr }\end{array}$ & $\begin{array}{c}\text { ooct } 03_{00011}, L 10 n 100 \\
\text { ooct } 03_{00018} \\
\text { ooct } 03_{00014}, L 12 n 1741\end{array}$ & $\begin{array}{l}4 \\
2 \\
3\end{array}$ & $\begin{array}{l}3 \\
3 \\
9\end{array}$ & 1 & qutrit Hesse SIC \\
\hline$\overline{4}$ & $\begin{array}{l}\operatorname{irr} \\
\operatorname{irr}\end{array}$ & $\begin{array}{l}\text { ooct } 04_{00058} \\
\text { ooct } 04_{00061}\end{array}$ & $\begin{array}{l}4 \\
3\end{array}$ & $\begin{array}{l}16 \\
16\end{array}$ & $\begin{array}{l}2 \\
2\end{array}$ & $\begin{array}{l}\text { 2-qubit IC } \\
\text { 2-qubit IC }\end{array}$ \\
\hline 5 & $\begin{array}{l}\operatorname{irr} \\
\operatorname{irr} \\
\operatorname{irr}\end{array}$ & $\begin{array}{c}\text { ooct } 05_{00092} \\
\text { ooct } 05_{00285} \\
\text { ooct } 05_{00098}, L 13 n 11257\end{array}$ & $\begin{array}{l}3 \\
2 \\
4\end{array}$ & $\begin{array}{l}25 \\
25 \\
25\end{array}$ & $\begin{array}{l}1 \\
1 \\
1\end{array}$ & $\begin{array}{l}\text { 5-dit IC } \\
\text { 5-dit IC } \\
\text { 5-dit IC }\end{array}$ \\
\hline 6 & $\begin{array}{l}\text { cyc } \\
\text { irr } \\
\text { irr }\end{array}$ & $\begin{array}{l}\text { ooct } 06_{06328} \\
\text { ooct } 06_{01972} \\
\operatorname{ooct} 06_{00471}\end{array}$ & $\begin{array}{l}5 \\
3 \\
4\end{array}$ & $\begin{array}{l}36 \\
36 \\
36\end{array}$ & $\begin{array}{l}2 \\
2 \\
2\end{array}$ & $\begin{array}{l}\text { 6-dit IC } \\
\text { 6-dit IC } \\
\text { 6-dit IC }\end{array}$ \\
\hline
\end{tabular}

TABLE 3. A few 3-manifolds $M^{3}$ found from subgroups of the fundamental group associated to the Whitehead link. For $d \geq 4$, only the $M^{3}$ 's leading to an IC are listed.

A two-qubit tetrahedral manifold. The tetrahedral three-manifold otet08 00007 is remarkable in the sense that it corresponds to the subgroup of index 4 of $G$ that allows the construction of the two-qubit IC-POVM. The corresponding hyperbolic polyhedron taken from SnapPy is shown in Fig. 4a. Of the 29 orientable tetrahedral manifolds with at most 8 tetrahedra, 20 are two-colorable and each of those has at most 2 cusps. The 4 three-manifolds (with at most 8 tetrahedra) identified in Table 2 belong to the 20's and the two-qubit tetrahedral manifold otet $08_{00007}$ is one with just one cusp [37, Table 1].

3.2. Three-manifolds pertaining to the Whitehead link. One could also identify the 3-manifold substructure of another universal object, viz the Whitehead link $L_{0}[33$.

The cardinality list corresponding to the Whitehead link group $\pi_{1}\left(L_{0}\right)$ is

$$
\eta_{d}\left(L_{0}\right)=\{1,3,6,17,22,79,94,412,616,1659 \ldots\},
$$

Table 3 shows that the identified 3-manifolds for index $d$ subgroups of $\pi_{1}\left(L_{0}\right)$ are aggregates of $d$ octahedra. In particular, one finds that the qutrit Hesse SIC can be built from ooct03 00014 and that the two-qubit IC-POVM may be buid from ooct $04_{00058}$. The hyperbolic polyhedron for the latter octahedral manifold taken from SnapPy is shown on Fig. 4b. The former octahedral manifold follows from the link $L 12 n 1741$ shown in Fig. 5a and the corresponding polyhedron taken from SnapPy is shown in Fig. 5b. 


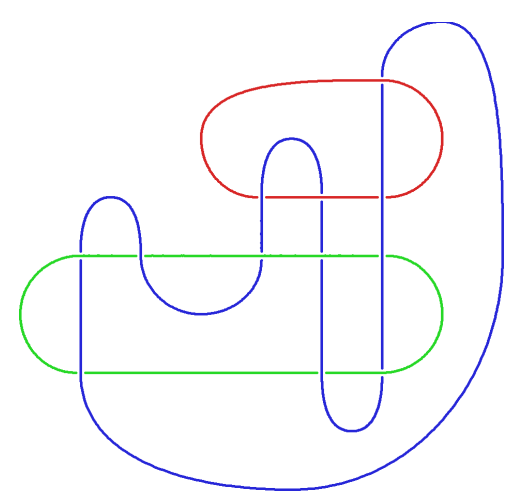

Link L12n1741

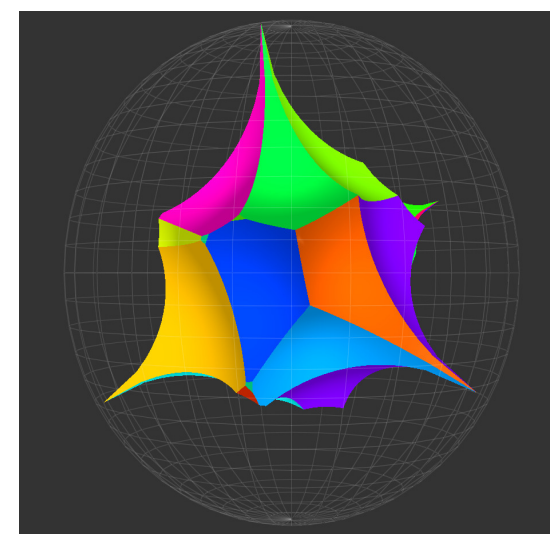

Manifold ooct03_00014

(a)

(b)

Figure 5. (a) The link $L 12 n 1741$ associated to the qutrit Hesse SIC, (b) The octahedral manifold ooct0300014 associated to the 2-qubit IC.

\begin{tabular}{|c|c|c|c|c|c|}
\hline d & ty & hom & $\mathrm{cp}$ & $M^{3}$ & comment \\
\hline 2 & $\begin{array}{l}\text { cyc } \\
. \\
.\end{array}$ & $\begin{array}{l}\frac{1}{2}+\frac{1}{2}+1+1+1 \\
\frac{1}{2}+1+1+1+1 \\
1+1+1+1+1\end{array}$ & $\begin{array}{l}3 \\
4 \\
5\end{array}$ & $\begin{array}{r}\text { ooct0 } 4_{00259} \\
\text { ooct04 } \\
00055 \\
{\operatorname{ooct} 04_{00048},} L 12 n 2226 \\
\end{array}$ & \\
\hline 3 & $\begin{array}{l}\text { cyc } \\
. \\
\text { irr } \\
. \\
\text {. }\end{array}$ & $\begin{array}{c}\frac{1}{3}+\frac{1}{3}+1+1+1 \\
\frac{1}{3}+1+1+1+1+1+1 \\
1+1+1+1+1+1+1+1 \\
1+1+1+1 \\
1+1+1+1+1+1 \\
1+1+1+1+1+1 \\
\end{array}$ & $\begin{array}{l}3 \\
5 \\
7 \\
4 \\
4 \\
5 \\
\end{array}$ & 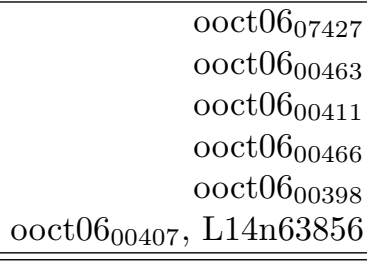 & $\begin{array}{l}\text { Hesse SIC } \\
\text { Hesse SIC }\end{array}$ \\
\hline 4 & $\begin{array}{l}\text { irr } \\
.\end{array}$ & $\begin{array}{c}\frac{1}{2}+\frac{1}{2}+1+1+1+1 \\
\frac{1}{2}+1+1+1+1+1+1\end{array}$ & $\begin{array}{l}4 \\
4\end{array}$ & $\begin{array}{r}\{63,300,10747 \cdots\} \\
\{127,2871,478956, \cdots\} \\
\end{array}$ & $\begin{array}{l}\text { 2QB MIC } \\
2 \mathrm{QB} \text { MIC }\end{array}$ \\
\hline
\end{tabular}

TABLE 4. Coverings of degrees 2 to 4 branched over the Borromean rings. The identification of the corresponding hyperbolic 3-manifold $M^{3}$ is at the 5th column. Only two types of 3-manifolds allow to build the Hesse SIC. The two 3 -manifolds of degree 4 allowing the construction of the twoqubit MIC are identified by the cardinality structure of their subgroups/coverings.

3.3. A few three-manifolds pertaining to Borromean rings. Three-manifolds corresponding to coverings of degree 2 and 3 of the 3 -manifold branched along the Borromean rings $L 6 a 4$ [that is a not a (3,3)-torus link but an hyperbolic link] (see Fig. 1c) are given in Table 4. The identified manifolds are hyperbolic octahedral manifolds of volume 14.655 (for the degree 2) and 21.983 (for the degree 3). 
MICHEL PLANAT $\dagger$, RAYMOND ASCHHEIM $\ddagger$ MARCELO M. AMARAL $\ddagger$ AND KLEE IRWIN $\ddagger$

\section{A FEW DeHn FILLINGS AND THEIR POVMs}

To summarize our findings of the previous section, we started from a building block, a knot (viz the trefoil knot $T_{1}$ ) or a link (viz the figure-of-eight knot $K_{0}$ ) whose complement in $S^{3}$ is a 3 -manifold $M^{3}$. Then a $d$-fold covering of $M^{3}$ was used to build a $d$-dimensional POVM, possibly an IC. Now we apply a kind of 'phase surgery' on the knot or link that transforms $M^{3}$ and the related coverings while preserving some of the POVMs in a way to be determined. We will start with our friend $T_{1}$ and arrive at a few standard 3-manifolds of historic importance, the Poincaré homology sphere [alias the Brieskorn sphere $\Sigma(2,3,5)$ ], the Brieskorn sphere $\Sigma(2,3,7)$ and a Seifert fibered toroidal manifold $\Sigma^{\prime}$. Then we introduce the 3-manifold $\Sigma_{Y}$ resulting from 0-surgery on the figure-of-eight knot 42. Later in this section, we will show how to use the $\{3,5,3\}$ Coxeter lattice and surgery to arrive at a hyperbolic 3 -manifold $\Sigma_{120 e}$ of maximal symmetry whose several coverings (and related POVMs) are close to the ones of the trefoil knot [38].

\begin{tabular}{|c|c|c|}
\hline $\mathrm{T}$ & Name & $\eta_{d}(T)$ \\
\hline$T_{1}$ & trefoil & 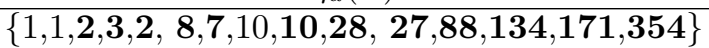 \\
\hline$T_{1}(-1,1)$ & $\Sigma(2,3,5)$ & $\{1,0,0,0, \mathbf{1}, \mathbf{1}, 0,0,0, \mathbf{1}, 0,1,0,0, \mathbf{1}\}$ \\
\hline$T_{1}(1,1)$ & $\Sigma(2,3,7)$ & $\{1,0,0,0,0,0, \mathbf{2}, 1, \mathbf{1}, 0,0,0,0,9,3\}$ \\
\hline$T_{1}(0,1)$ & $\Sigma^{\prime}$ & $\{1,1, \mathbf{2}, \mathbf{2}, 1, \mathbf{5}, \mathbf{3}, 2,4,1,1,12,3,3, \mathbf{4}\}$ \\
\hline$K_{0}(0,1)$ & $\Sigma_{Y}$ & $\{1,1,1, \mathbf{2}, 2, \mathbf{5}, 1,2,2,4, \mathbf{3}, 17,1,1,2\}$ \\
\hline$v_{2413}(-3,2)$ & $\Sigma_{120 e}$ & $\{1,1, \mathbf{1}, \mathbf{4}, 1, \mathbf{7}, 2,25, \mathbf{3}, \mathbf{1 0}, \mathbf{1 0}, \mathbf{6 2}, 1,30,23\}$ \\
\hline
\end{tabular}

TABLE 5. A few surgeries (column 1), their name (column 2) and the cardinality list of $d$-coverings (alias conjugacy classes of subgroups). Plain characters are used to point out the possible construction of an IC-POVM in at least one the corresponding three-manifolds (see [2] and Sec. 2 for the ICs corresponding to $T_{1}$ ).

Let us start with a Lens space $L(p, q)$ that is 3-manifold obtained by gluing the boundaries of two solid tori together, so that the meridian of the first solid torus goes to a $(p, q)$-curve on the second solid torus [where a $(p, q)$-curve wraps around the longitude $p$ times and around the meridian $q$ times]. Then we generalize this concept to a knot exterior, i.e. the complement of an open solid torus knotted like the knot. One glues a solid torus so that its meridian curve goes to a $(p, q)$-curve on the torus boundary of the knot exterior, an operation called Dehn surgery [1, p. 275],[26, p 259], [39]. According to Lickorish's theorem, every closed, orientable, connected 3-manifold is obtained by performing Dehn surgery on a link in the 3-sphere.

\subsection{A few surgeries on the trefoil knot.}

The Poincaré homology sphere. The Poincaré dodecahedral space (alias the Poincaré homology sphere) was the first example of a 3-manifold not the 3 -sphere. It can be obtained from $(-1,1)$ surgery on the trefoil knot $T_{1}[40$.

Let $p, q, r$ be three positive integers and mutually coprime, the Brieskorn sphere $\Sigma(p, q, r)$ is the intersection in $\mathbb{C}^{3}$ of the 5 -sphere $S^{5}$ with the surface of equation $z_{1}^{p}+z_{2}^{q}+z_{3}^{r}=1$. The homology of a Brieskorn sphere is that of the sphere $S^{3}$. A Brieskorn sphere is homeomorphic but not diffeomorphic to $S^{3}$. The sphere $\Sigma(2,3,5)$ may be identified to the Poincaré homology sphere. The sphere $\Sigma(2,3,7)$ [42] may be obtained from $(1,1)$ surgery on $T_{1}$. Table 5 provides the sequences $\eta_{d}$ 
for the corresponding surgeries $( \pm 1,1)$ on $T_{1}$. Plain digits in these sequences point out the possibility of building ICs of the corresponding degree. This corresponds to a considerable filtering of the ICs coming from $T_{1}$.

For instance, the smallest IC from $\Sigma(2,3,5)$ has dimension five and is precisely the one coming from the congruence subgroup $5 A^{0}$ in Table 1 . But it is built from a non modular (fundamental) group whose permutation representation of the cosets is the alternating group $A_{5} \cong\langle(1,2,3,4,5),(2,4,3)\rangle$ (compare [12, Sec. 3.3]).

The smallest dimensional IC derived from $\Sigma(2,3,7)$ is 7 -dimensional and twovalued, the same than the one arising from the congruence subgroup $7 A^{0}$ given in Table 1. But it arises from a non modular (fundamental) group with the permutation representation of cosets as $P S L(2,7) \cong\langle(1,2,4,6,7,5,3),(2,5,3)(4,6,7)\rangle$.

4.2. The Seifert fibered toroidal manifold $\Sigma^{\prime}$. An hyperbolic knot (or link) in $S^{3}$ is one whose complement is 3-manifold $M^{3}$ endowed with a complete Riemannian metric of constant negative curvature, i.e. it has a hyperbolic geometry and finite volume. A Dehn surgery on a hyperbolic knot is exceptional if it is reducible, toroidal or Seifert fibered (comprising a closed 3-manifold together with a decomposition into a disjoint union of circles called fibers). All other surgeries are hyperbolic. These categories are exclusive for a hyperbolic knot. In contrast, a non hyperbolic knot such as the trefoil knot admits a toroidal Seifert fiber surgery $\Sigma^{\prime}$ obtained by $(0,1)$ Dehn filling on $T_{1}[41$.

The smallest dimensional ICs built from $\Sigma^{\prime}$ are the Hesse SIC that is obtained from the congruence subgroup $\Gamma_{0}(2)$ (as for the trefoil knot) and the two-qubit IC that comes from a non modular fundamental group [with cosets organized as the alternating group $\left.A_{4} \cong\langle(2,4,3),(1,2,3)\rangle\right]$.

4.3. Akbulut's manifold $\Sigma_{Y}$. Exceptional Dehn surgery at slope $(0,1)$ on the figure-of-eight knot $K_{0}$ leads to a remarkable manifold $\Sigma_{Y}$ found in [42] in the context of 3-dimensional integral homology spheres smoothly bounding integral homology balls. Apart from its topological importance, we find that some of its coverings are associated to already discovered ICs and those coverings have the same fundamental group $\pi_{1}\left(\Sigma_{Y}\right)$.

The smallest IC-related covering (of degree 4 ) occurs with integral homology $\mathbb{Z}$ and the congruence subgroup $\Gamma_{0}(3)$ also found from the trefoil knot (see Table 1). Next, the covering of degree 6 and homology $\frac{\mathbb{Z}}{5}+\mathbb{Z}$ leads to the 6 -dit IC of type $3 C^{0}$ (also found from the trefoil knot). The next case corresponds to the (non-modular) 11-dimensional (3-valued) IC.

4.4. The hyperbolic manifold $\Sigma_{120 e}$. The hyperbolic manifold closest to the trefoil knot manifold known to us was found in [38. The goal in [38] is the search of -maximally symmetric- fundamental groups of 3-manifolds. In two dimensions, maximal symmetry groups are called Hurwitz groups and arise as quotients of the $(2,3,7)$-triangle groups. In three dimensions, the quotients of the minimal co-volume lattice $\Gamma_{\min }$ of hyperbolic isometries, and of its orientation preserving subgroup $\Gamma_{\text {min }}^{+}$, play the role of Hurwitz groups. Let $C$ be the $\{3,5,3\}$ Coxeter group, $\Gamma_{\min }$ the split extension $C \rtimes \mathbb{Z}_{2}$ and $\Gamma_{\min }^{+}$one of the index two subgroups of $\Gamma_{\min }$ of presentation

$$
\Gamma_{\text {min }}^{+}=\left\langle x, y, z \mid x^{3}, y^{2}, z^{2},(x y z)^{2},(x z y z)^{2},(x y)^{5}\right\rangle .
$$

According to [38, Corollary 5], all torsion-free subgroups of finite index in $\Gamma_{m i n}^{+}$ have index divisible by 60 . There are two of them of index 60 , called $\Sigma_{60 a}$ and $\Sigma_{60 b}$, obtained as fundamental groups of surgeries $m 017(-4,3)$ and $m 016(-4,3)$. Torsion-free subgroups of index 120 in $\Gamma_{\min }^{+}$are given in Table 6. It is remarkable 
MICHEL PLANAT $\dagger$, RAYMOND ASCHHEIM $\ddagger$, MARCELO M. AMARAL $\ddagger$ AND KLEE IRWIN $\ddagger$

that these groups are fundamental groups of oriented three-manifolds built with a single icosahedron except for $\Sigma_{120 e}$ and $\Sigma_{120 g}$.

\begin{tabular}{|c|c|c|}
\hline manifold $\mathrm{T}$ & "subgroup & $\overline{\overline{\eta_{d}(T)}}$ \\
\hline oicocld01 $00001=s$ & $\Sigma_{1}$ & $\{1,0,0,0,0,8,2,1,1,8\}$ \\
\hline $01_{00000}=s 900(-3,2)$ & $\Sigma_{12}$ & $\{1,0,0,0,5,8,10,15,5,24\}$ \\
\hline oicocld $01_{00003}=v 2051(-3,2)$ & $\Sigma_{120 c}$ & $\{1,0,0,0,0,4,8,12,6,6\}$ \\
\hline $\mathrm{d} 01_{00002}=s 890(3,2)$ & $\Sigma_{12}$ & $\{1,0,1,5,0,9,0,35,9,2\}$ \\
\hline$v 2413(-3,2) \neq{\text { oicocld } 01_{00004}}$ & $\Sigma_{120 e}$ & $\{1,1,1,4,1,7,2,25,3,10\}$ \\
\hline $\mathrm{d} 01_{00005}=v 3215(1,2)$ & $\Sigma_{120 f}$ & $\{1,0,0,0,0,14,10,5,10,17\}$ \\
\hline v3318(-1,2) & $\Sigma_{120 g}$ & $\{1,3,1,2,0,11,0,23,12,14\}$ \\
\hline
\end{tabular}

TABLE 6 . The index 120 torsion free subgroups of $\Gamma_{\min }^{+}$and their relation to the single isosahedron 3 -manifolds [38]. The icosahedral symmetry is broken for $\Sigma_{120 e}$ (see the text for details).

The group $\Sigma_{120 e}$ is special in the sense that many small dimensional ICs may be built from it in contrast to the other groups in Table 6 . The smallest ICs that may be build from $\Sigma_{120 e}$ are the Hesse SIC coming from the congruence subgroup $\Gamma_{0}(2)$, the two-qubit IC coming the congruence subgroup $4 A^{0}$ and the 6 -dit ICs coming from the congruence subgroups $\Gamma(2), 3 C^{0}$ or $\Gamma_{0}(4)$ (see 2 , Sec. 3 and Table 1]). Higher dimensional ICs found from $\Sigma_{120 e}$ does not come from congruence subgroups.

\section{Conclusion}

The relationship between 3-manifolds and universality in quantum computing has been explored in this work. Earlier work of the first author already pointed out the importance of hyperbolic geometry and the modular group $\Gamma$ for deriving the basic small dimensional IC-POVMs. In Sec. 2, the move from $\Gamma$ to the trefoil knot $T_{1}$ (and the braid group $B_{3}$ ) to non hyperbolic 3 -manifolds could be investigated by making use of the $d$-fold coverings of $T_{1}$ that correspond to $d$-dimensional POVMs (some of them being IC). Then, in Sec. 3, we passed to universal links (such as the figure-of-eight knot, Whitehead link and Borromean rings) and the related hyperbolic platonic manifolds as new models for quantum computing based POVMs. Finally, in Sec. 4, Dehn fillings on $T_{1}$ were used to explore the connection of quantum computing to important exotic 3 -manifolds [i.e. $\Sigma(2,3,5)$ and $\Sigma(2,3,7)$ ], to the toroidal Seifert fibered $\Sigma^{\prime}$, to Akbulut's manifold $\Sigma_{Y}$ and to a maximum symmetry hyperbolic manifold $\Sigma_{120 e}$ slightly breaking the icosahedral symmetry. It is expected that our work will have importance for new ways of implementing quantum computing and for the understanding of the link between quantum information theory and cosmology [43, 44, 45].

\section{AUthor CONTRIBUTIONS STATEMENT}

All authors contributed significantly to the subject and content of the paper. M. P. wrote the manuscript and the co-authors rewiewed it.

\section{FUNDING}

The first author acknowledges the support by the French "Investissements d'Avenir" program, project ISITE-BFC (contract ANR-15-IDEX-03). The other ressources came from Quantum Gravity Research. 


\section{Competing Interests}

The authors declare no competing interests, financial and non-financial.

\section{REFERENCES}

[1] W. P. Thurston, Three-dimensional geometry and topology (vol. 1), (Princeton University Press, Princeton, 1997).

[2] M. Planat, The Poincaré half-plane for informationally complete POVMs, Entropy 2016 (2018).

[3] H. M. Hilden, M. T. Lozano, J. M. Montesinos and W. C. Whitten, On universal groups and three-manifolds, Inventiones Mathematicae 87 441-45 (1987).

[4] E. Fominikh, S. Garoufalidis, M. Goerner, V. Tarkaev and A. Vesnin, A census of tethahedral hyperbolic manifolds, Exp. Math. 25 466-481 (2016).

[5] A. Yu Kitaev, Fault-tolerant quantum computation by anyons, Annals Phys. 303 2-30 (2003).

[6] C. Nayak, S. H. Simon, A. Stern, M. Freedman and S. Das Sarma, Non-Abelian anyons and topological quantum computation, Rev. Mod. Phys. 801083 (2008).

[7] Z. Wang, Topological Quantum Computation, (American Mathematical Soc., Rhode Island, Number 112, 2010).

[8] J K. Pachos, Introduction to Topological Quantum Computation (Cambridge University Press, Cambridge, 2012).

[9] S. Vijay and L. Fu, A generalization of non-abelian anyons in three dimensions, arXiv 1706.07070 (2017).

[10] S. Bravyi and A. Kitaev, Universal quantum computation with ideal Clifford gates and noisy ancillas, Phys. Rev. A71 022316 (2005).

[11] M. Planat and R. Ul Haq, The magic of universal quantum computing with permutations, Advances in mathematical physics 217, ID 5287862 (2017) 9 pp.

[12] M. Planat and Z. Gedik, Magic informationally complete POVMs with permutations, R. Soc. open sci. 4170387 (2017).

[13] L. H. Kauffman and R. L. Baadhio, Quantum topology (Series on knots and everything), (World Scientific, 1993).

[14] L. H. Kauffman, Knot logic and topological quantum computing with Majorana fermions, in Linear and algebraic structures in quantum computing, (J. Chubb, A. Eskandarian and V. Harizanov eds, Lecture notes in logic 45, Cambridge Univ. Press, 2016).

[15] N. Seiberg, T. Senthil, C. Wang and E. Witten, A duality web in $2+1$ dimensions and condensed matter physics, Ann. Phys. 374 395-433 (2016).

[16] D. Gang, Y. Tachikawa and K. Yonekura, Smallest $3 d$ hyperbolic manifolds via simple $3 d$ theories, Phys. Rev. D 96 061701(R) (2017).

[17] N. C. Lim and S. E. Jackson, Molecular knots in biology and chemistry, J. Phys. Condens. Matter 27354101 (2015).

[18] K. Irwin, Toward a unification of physics and number theory, https://www.researchgate.net/publication/314209738 _Toward_the_Unification_of_Physics_and_Number_Theory

[19] J. Milnor, The Poincaré conjecture 99 years later: a progress report (The Clay Mathematics Institute 2002 Annual Report, 2003), http://www.math.sunysb.edu/ jack/PREPRINTS/poiproof.pdf, Retrieved 2018-01-01.

[20] M. Planat, On the geometry and invariants of qubits, quartits and octits, Int. J. Geom. Methods in Mod. Phys. 8 303-313 (2011).

[21] N. S. Manton, Connections on discrete fiber bundles, Commun. Math. Phys. 113 341-351 (1987).

[22] R. Mosseri and R. Dandoloff, Geometry of entangled states, Bloch spheres and Hopf fibrations, it J. Phys. A: Math. Gen. 3410243 (2001).

[23] J. A. Nieto, Division-Algebras/Poincare-Conjecture Correspondence, J. Mod. Phys. (Scientific Research) 4 32-36 (2013).

[24] A. Sen, R. Aschheim and K. Irwin, Emergence of an aperiodic Dirichlet space from the tetrahedral units of an icosahedral internal space Mathematics 529 (2017). 
M6ICHEL PLANAT $\dagger$, RAYMOND ASCHHEIM $\ddagger$, MARCELO M. AMARAL $\ddagger$ AND KLEE IRWIN $\ddagger$

[25] F. Fang, D. Hammock and K. Irwin, Methods for calculating empires in quasicrystals, Crystals 7304 (1997).

[26] C. C. Adams, The knot book, An elementary introduction to the mathematical theory of knots (W. H. Freeman and Co, New York, 1994).

[27] A. D. Mednykh, A new method for counting coverings over manifold with fintely generated fundamental group, Dokl. Math. 74 498-502 (2006).

[28] M. Culler, N. M. Dunfield, M. Goerner, and J. R. Weeks, SnapPy, a computer program for studying the geometry and topology of 3-manifolds, http://snappy.computop.org.

[29] H. M. Hilden, M. T. Lozano and J. M. Montesinoos, On knots that are universal, Topology 24 499-504 (1985).

[30] Chris A. Fuchs, On the quantumness of a Hibert space, Quant. Inf. Comp. 4 467-478 (2004).

[31] M. Appleby, T. Y. Chien, S. Flammia and S. Waldron, Constructing exact symmetric informationally complete measurements from numerical solutions, Preprint 1703.05981 [quant-ph].

[32] D. Rolfsen, Knots and links (Mathematics Lecture Series 7, Houston, 1990).

[33] D. Gabai, The Whitehead manifold is a union of two Euclidean spaces, J. Topol. 4 529-534 (2011).

[34] J. Milnor, On the 3-dimensional Brieskorn manifolds $M(p, q, r)$, in Knots, groups and 3-manifolds (ed. L. P. Neuwirth), Annals of Math. Study 84, Princeton Univ. Press, Princeton, 175-225 (1975).

[35] J. Hempel, The lattice of branched covers over the Figure-eight knot, Topol. Appl. 34 183-201 (1990).

[36] R. C. Haraway, Determining hyperbolicity of compact orientable 3-manifolds with torus boundary, Arxiv 1410.7115 [math.GT].

[37] S. A. Ballas, J. Danciger and G. S. Lee, Convex projective structures on nonhyperbolic three-manifolds, Arxiv 1508.04794 [math.GT].

[38] M. Conder, G. Martin and A. Torstensson, Maximal symmetry groups of hyperbolic 3-manifolds New Zealand J. Math. 353762 (2006).

[39] C. M. Gordon, Dehn filling: a survey, Knot Theory, Banach Center Publ. 42 129-144, Polish Acad. Sci., Warsaw (1998).

[40] R. C. Kirby and M. G. Scharlemann, Eight faces of the Poincaré homology 3-sphere, in Geometric Topology (Acad. Press, New York, 1979) pp 113-146.

[41] Ying-Qing Wu, Seifert fibered surgery on Montesinos knots, Arxiv 1207.0154 [math.GT], to appear in Comm. Anal. Geom.

[42] S. Akbulut and K. Larson, Brieskorn spheres bounding rational balls, ArXiv 1704.07739 [math.GT].

[43] K. T. Chan, H. Zainuddin, K. A. M. Atan and A. A. Siddig, Computing Quantum Bound States on Triply Punctured Two-Sphere Surface, Chin. Phys. Lett. 33090301 (2016).

[44] R. Aurich, F. Steiner and H. Then, Numerical computation of Maass waveforms and an application to cosmology, in Hyperbolic Geometry and Applications in Quantum Chaos and Cosmology (Jens Bolte and Frank Steiner eds, Cambridge Univ. Press, 2012), Preprint gr-qc/0404020.

[45] T. Asselmeyer-Maluga, Smooth quantum gravity: Exotic smoothness and Quantum gravity, in At the Frontier of Spacetime Scalar-Tensor Theory, Bells Inequality, Machs Principle, Exotic Smoothness (Fundamental Theories of Physics book series (FTP), T. Asselmeyer-Maluga ed. (2016), pp 247-308).

† Université de Bourgogne/Franche-Comté, Institut FEMTO-ST CNRS UMR 6174, 15 B Avenue des Montboucons, F-25044 Besançon, France.

E-mail address: michel.planat@femto-st.fr 
UNIVERSAL QUANTUM COMPUTING AND THREE-MANIFOLDS

$\ddagger$ Quantum Gravity Research, Los Angeles, CA 90290, USA

E-mail address: raymond@QuantumGravityResearch.org

E-mail address: Klee@quantumgravityresearch.org

E-mail address: Marcelo@quantumgravityresearch.org 